\title{
Mayotte s'ancre dans la république française
}

Un contre-sens de l'histoire?

\section{François Taglioni}

\section{OpenEdition}

Journals

Édition électronique

URL : http://journals.openedition.org/echogeo/11277

DOI : 10.4000/echogeo.11277

ISSN : 1963-1197

\section{Éditeur}

Pôle de recherche pour l'organisation et la diffusion de l'information géographique (CNRS UMR 8586)

\section{Référence électronique}

François Taglioni, « Mayotte s'ancre dans la république française », EchoGéo [En ligne], Sur le Vif, mis en ligne le 01 juillet 2009, consulté le 01 mai 2019. URL : http://journals.openedition.org/ echogeo/11277; DOI : 10.4000/echogeo.11277

Ce document a été généré automatiquement le 1 mai 2019.

\section{(c) (i) (9)}

EchoGéo est mis à disposition selon les termes de la licence Creative Commons Attribution - Pas d'Utilisation Commerciale - Pas de Modification 4.0 International 


\section{Mayotte s'ancre dans la république française}

Un contre-sens de l'histoire?

François Taglioni

1 L'outre-mer français est à l'honneur dans les médias ces derniers mois pour ses crises sociales, identitaires, politiques voire institutionnelles. Après les remous qui ont agité Mayotte en mai 2008 suite à l'extradition du président déchu d'Anjouan (Taglioni, 2008), La Réunion en novembre 2008 où les transporteurs ont paralysé l'île pour protester contre le prix du carburant, La Guyane en novembre/décembre 2008 contre l'essence chère, c'est au tour des Antilles françaises et de La Réunion de faire la une de l'actualité en janvier et février 2009. Ces mouvements d'instabilité politique et économique, qui risque notamment de se poursuivre avec le référendum d'autodétermination pour l'indépendance en Nouvelle-Calédonie entre 2014 et 2018, se double d'un désir contradictoire d'intégration à la métropole qui a poussé la collectivité territoriale de Mayotte vers la départementalisation au sein de la République française. C'est sans doute un paradoxe de l'histoire que de voir une ancienne colonie se battre pour devenir le $101^{\mathrm{e}}$ département français et le $5^{\text {e }}$ département d'outre-mer (DOM).

2 Pour essayer de saisir les enjeux et les perspectives de cette départementalisation, il est utile de revenir sur la complexité des statuts institutionnels à la carte des outre-mers français, de replacer Mayotte dans son environnement archipélagique et enfin de se poser la question de la spécificité éventuelle des outre-mers français au regard des outre-mers liés à d'autres métropoles dans le monde.

\section{La situation institutionnelle de Mayotte et des outre- mers}

3 L'outre-mer français est d'un point de vue institutionnel (La documentation française, 2008) d'une assez grande complexité puisque l'on dénombre, depuis la révision 
constitutionnelle du 28 mars 2003 et des lois de février 2007, trois grandes catégories de statuts qui sont régis par deux régimes législatifs.

4 La première catégorie est celle des régions monodépartementales d'outre-mer que l'on appelle communément les DOM (départements d'outre-mer) bien que ce soit aussi des ROM (régions d'outre-mer). Il s'agit de La Guadeloupe, de La Martinique et de La Guyane, qui constituent les départements français d'Amérique (DFA), et de La Réunion. La possibilité de fusionner département et région leur est donnée. Depuis le référendum du 29 mars 2009 Mayotte, anciennement collectivité départementale d'outre-mer va devenir dans un proche avenir un DOM. Cette catégorie passera donc à 5 membres.

5 La deuxième, assez hétéroclite, est celle des collectivités d'outre-mer (COM). On y retrouve Saint-Pierre-et-Miquelon dans l'Atlantique nord, Saint-Martin et Saint-Barthélemy dans les Petites Antilles, Wallis et Futuna dans le Pacifique sud et enfin La Polynésie française également dans le Pacifique sud.

6 La troisième, dite des collectivités sui generis est tout aussi hétéroclite puisqu'elle rassemble d'une part La Nouvelle-Calédonie dans le Pacifique sud et d'autre part les Terres australes et antarctiques françaises (TAAF) auxquelles on rattache les îles éparses du canal du Mozambique.

7 Les deux régimes législatifs qui s'appliquent à ces outre-mers sont le régime de l'identité législative (article 73 de la Constitution) et le régime de spécialité législative et d'autonomie (article 74 de la Constitution). Le premier s'applique au DOM-ROM et il indique que les lois et règlements nationaux sont applicables de plein droit avec des adaptations possibles en fonction des contextes locaux. Le second est celui qui définit le statut particulier de chaque collectivité qu'elle soit d'outre-mer ou sui generis.

Cette mise au point est un peu technique mais nécessaire car elle permet de bien définir les outre-mers français et de rappeler que ce qui fut autrefois des colonies, puis des DOMTOM sont aujourd'hui des territoires avec des statuts à la carte qui traduisent les adaptions du législateur vis-à-vis de l'outre-mer français. Ces ajustements présentent néanmoins le risque du particularisme à tout prix et de la difficulté aux gouvernements successifs de trouver des solutions globales aux difficultés de l'outre-mer. Les états généraux entamés en avril 2009 le démontrent.

9 Le statut de Mayotte est un bel exemple de ces évolutions statutaires puisque l'île fut tour à tour colonie française à partir de 1841, territoire d'outre-mer à partir de 1946 (en association avec les autres îles de l'archipel des Comores), collectivité territoriale à partir de 1976, collectivité départementale depuis 2001 et enfin département d'outre-mer sans doute en 2011. Le passage le plus délicat de cette évolution, et aussi le plus lourd de conséquences aujourd'hui encore, est le rejet par les Mahorais de l'indépendance telle que souhaitée par les trois autres îles de l'archipel des Comores : Anjouan, Grande Comore et Mohéli. De fait, la population mahoraise avait répondu «non » à plus de $60 \%$ des votants, le 22 décembre 1974, au premier référendum d'autodétermination des Comores. Lors d'un deuxième référendum le 8 février 1976, les Mahorais réitèrent avec force et vigueur leur souhait, à plus de $99 \%$ des votants, de demeurer au sein de la République Française. Les Comores deviennent donc indépendantes à trois le 6 juillet 1975 et Mayotte reste dans le giron de la République française (figure 1). 
Figure 1 - L'Union des Comores et Mayotte

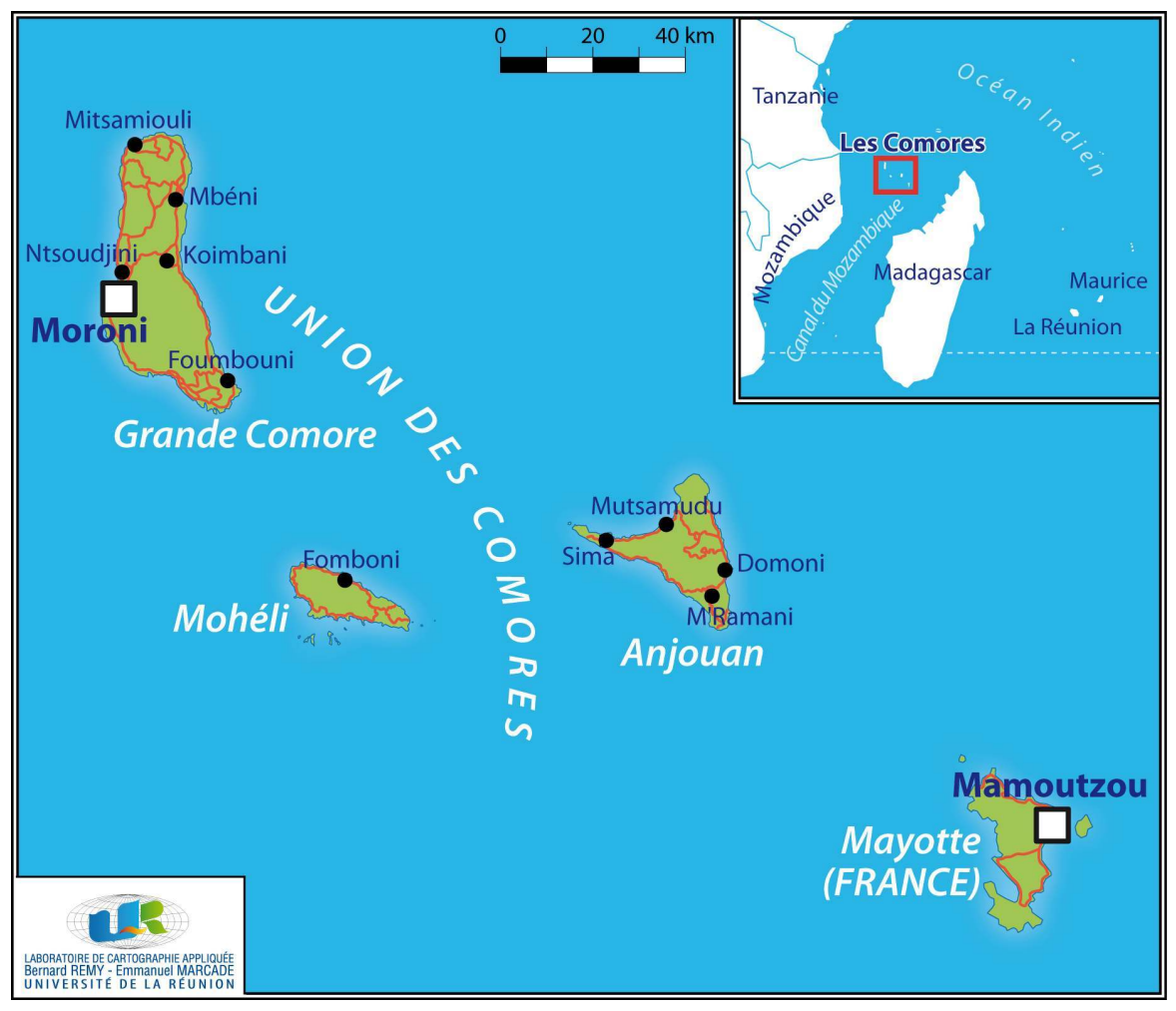

Dans les Antilles françaises les évolutions statutaires sont plus simples, pour le moment, puisque de colonies en 1635 La Martinique et l'archipel de La Guadeloupe deviennent départements français d'outre-mer en 1946 puis régions d'outre-mer monodépartementales en 1984. La révision de la constitution française en 2003 leur donne la possibilité de fusionner département et région en une seule entité. L'identité législative (article 73) est de mise avec la possibilité d'élaborer des règlements. Le référendum du 7 décembre 2003 consacre le statu quo institutionnel dans l'archipel de La Guadeloupe et de La Martinique mais donne à Saint-Barthélemy et Saint-Martin, jusque là communes de l'archipel de La Guadeloupe, une plus grande souveraineté et la qualité de collectivité d'outre-mer en devenir (depuis juillet 2007) avec le régime de spécialité législative et l'autonomie. Deux nouvelles collectivités se sont donc ajoutées à la liste déjà longue des outre mers français.

11 Aujourd'hui et dans les années à venir, la question de l'autonomie des Antilles françaises continuera à être posée. La possibilité du passage à l'article 74 pour répondre à la grave crise sociale, identitaire et économique du début de l'année 2009 est très présente dans l'espace public et surtout l'espace politique. La crise actuelle est elle-même le fruit de plusieurs siècles d'une géographie et d'une histoire complexes entre la métropole et les Antilles. Il faudrait cependant qu'un nouveau vote populaire entérine par référendum cette volonté d'autonomie dans le cadre de l'article 74 qui est certes déjà adopté par le congrès des élus de Martinique le 18 décembre 2008. Le président Sarkozy en visite à La Martinique le 26 juin 2009 a fait la promesse d'un nouveau référendum sur l'autonomie en posant comme hypothèse que " les esprits ont évolué » depuis décembre 2003. Il reste donc à savoir si le peuple martiniquais est effectivement prêt à faire passer cette fois le « oui » qui avait fait défaut le 7 décembre 2003 et si les élus locaux de Martinique se sentent en position d'assumer de nouvelles prérogatives législatives et exécutives avec les 
droits et devoirs que cela suppose. Une solution intermédiaire serait celle d'une assemblée unique sous le régime de l'article 73.

Pour le moment, le congrès guadeloupéen, lui, reste conforme au vote populaire du référendum de 2003 (ou le non avait été très largement plébiscité contrairement à La Martinique ; 72,98 \% de non en Guadeloupe et 50,48 \% en Guadeloupe) et ne demande pas à s'associer au processus d'évolution statutaire. La position des élus et du peuple de la Guadeloupe peut sembler paradoxale eu égard à l'énergie qu'ils ont déployé pendant des semaines de grève générale en ce début d'année 2009. La revendication première en Guadeloupe n'était pas celle de l'autonomie mais bien plutôt celle de l'impasse économique des plus démunis et d'une lutte identitaire face à une minorité blanche qui détient toujours les clés de la finance et du foncier sur l'archipel et aussi face à une métropole française qui a parfois du mal à prendre la mesure des problématiques locales qu'elles soient économiques, sociales ou politiques.

Pour La Réunion et La Guyane, l'histoire coloniale et institutionnelle est sensiblement la même que pour les Antilles mais les évolutions récentes diffèrent puisque les habitants de ces deux départements d'outre-mer n'ont pas pris part au référendum du 7 décembre 2003. Il semble bien que ni la classe politique ni le peuple réunionnais ne désirent un approfondissement de leur autonomie vis-à-vis de la métropole. A ce titre, La Réunion est même en retrait par rapport aux départements français d'Amérique (DFA) puisqu'elle bénéficie de l'identité législative mais sans possibilité d'élaborer des règlements. En revanche, le congrès de la Guyane, comme celui de La Martinique, a récemment demandé une possible évolution statutaire. Un référendum, à l'instar de celui proposé pour La Martinique, est sans doute souhaitable

\section{Les enjeux de la départementalisation à Mayotte}

14 Il semblerait que les évolutions statutaires de Mayotte se soient accompagnées à chaque étape d'une amélioration du développement humain. Certes Mayotte n'est pas encore aujourd'hui au niveau de La Réunion, département d'outre-mer, et encore moins de celui de la métropole mais néanmoins les indicateurs classiques du développement vont dans la bonne direction. C'est le cas de l'espérance de vie, du taux de mortalité infantile, de l'alphabétisation, ou encore de la scolarisation. Ces quelques indicateurs apparaissent d'autant meilleurs que ceux de ses proches voisins, Anjouan, Grande Comore, Mohéli ou Madagascar, sont bien plus médiocres. Néanmoins cette relative prospérité est à nuancer si on la met en perspective avec les standards des pays les plus avancés. On constate alors que Mayotte, en dépit d'une croissance économique de l'ordre de $9 \%$ par an, souffre d'un déficit économique d'ordre structurel qui se traduit par des carences en matière de santé, un fort taux de chômage (environ 22 \% de la population active) et notamment des jeunes, un revenu annuel moyen inférieur de près de $70 \%$ de celui de la métropole ( 9300 euros contre 30000 euros), des infrastructures sociales parfois défaillantes, un bâti précaire dominant, une transition démographique loin d'être achevée avec une forte natalité et une faible mortalité (les moins de 20 ans constituent $54 \%$ de la population totale en 2007 selon l'Insee et le nombre d'enfants par femme est de 3,4). Ces quelques éléments soulignent la fragilité d'une économie largement dépendante des transferts financiers métropolitains. Dans ce contexte, les enjeux de la départementalisation sont de taille pour Mayotte. 
15 Le référendum du 29 mars 2009 donne en effet à Mayotte les clés de la départementalisation à l'horizon 2011. Les $95 \%$ de votes pour le « oui » sont néanmoins à relativiser au regard du taux d'abstention qui avoisinait les $40 \%$, Selon toute vraisemblance, Mayotte ne sera pas une région monodépartementale comme les 4 autres DOM mais plutôt une fusion des deux, un DROM (département et région d'outre-mer avec une assemblée unique). Pour le président et les élus de cette assemblée unique il s'agira dans les années à venir de relever nombre de défis économiques, politiques et sociaux en accord avec le pacte pour la départementalisation définit par le gouvernement français (cf. bibliographie). En effet, le passage de l'article 74 à l'article 73 suppose un certain nombre de changements plus ou moins conséquents pour les populations mahoraises. Il y aura de nouveaux droits mais aussi de nouveaux devoirs. Il serait fastidieux d'entrer dans les détails juridiques et techniques de ce que seront dans le détail ces évolutions, d'autant que de nombreux paramètres sont encore à définir, mais d'ores et déjà on peut établir les grandes lignes des enjeux de la départementalisation.

16 Les répercussions économiques et sociales seront bien sûr au centre de toutes les attentions tant la situation est fragile à Mayotte. L'économie mahoraise s'appuie sur quelques secteurs. L'agriculture est largement destinée à l'autosubsistance de ses cultivateurs et seuls deux produits d'exportation, la vanille et l'ylang-ylang, fournissent des devises. Le tourisme est un secteur qui stagne et qui se limite à moins de 40000 touristes par an. En dépit d'une phase de croissance ces dernières années, il reste prisonnier d'une desserte aérienne limitée ainsi que des risques sanitaires qui peuvent le faire chuter comme cela a été le cas avec l'épidémie de chikungunya en 2007. Le secteur productif est très limité et au total les exportations représentent moins de $2 \%$ des importations. Le climat social est souvent tendu et pour le seul mois de mai 2009 plusieurs grèves dénotent des tensions économiques et leurs répercussions sociales dans l'île. Ainsi, les instituteurs ont fait grève deux jours pour que soit mis en place un système de préférence locale pour le recrutement des instituteurs d'Etat recrutés à Mayotte. Cela constitue bien sûr un paradoxe à la départementalisation qui prévoit l'alignement le plus large possible des lois de la république française à Mayotte. Par ailleurs, depuis le 11 mai la moitié (une quinzaine) des agents du comité de tourisme sont également en grève car pèse une menace de licenciement et une difficulté à boucler le budget de l'année 2009 faute de subvention suffisante du Conseil général. Enfin, les agents de la firme Total sont eux aussi en grève illimitée depuis le 18 mai 2009 pour des questions de rémunérations et cela n'est pas sans rappeler la situation délicate et tendue qu'a connu Mayotte en février 2008 avec une distribution d'essence des plus limitées sur fond de grève de plusieurs semaines à la poste et dans les écoles primaires.

17 A cette instabilité sociale s'ajoute un flou juridique sur un certain nombre de dossiers incompatibles avec un statut de département français. Ainsi, la mise en place d'un état civil (absence des noms de famille), qui fait actuellement défaut et qui symbolise le passage du droit coranique au droit commun, est devenue un chantier prioritaire pour le Conseil général avec toutes les difficultés inhérentes à cette entreprise. Par ailleurs, la justice cadiale, aujourd'hui rendue par l'un des 22 magistrats musulmans appelés les cadis, qui occupent des fonctions civiles, judiciaires, notariales et religieuses, sera de moins en moins possible et légale. Il est à noter qu'actuellement les cadis sont des agents territoriaux relevant du conseil général. En ce qui concerne la polygamie, toujours pratiquée bien qu'interdite depuis 2005, elle est sommée de disparaître définitivement ; ainsi les mariages polygames seront désormais interdits. De la même façon, les écoles 
coraniques devront trouver leur place dans une île laïque. En ce qui concerne l'âge légal minimum du mariage, il sera désormais fixé à 18 ans au lieu de 15 ans et entraînera la disparition du Wali (tuteur matrimonial). D'autre part, les Mahorais seront progressivement soumis à divers impôts et notamment immobiliers (taxe foncière, taxe d'habitation) en lien avec le cadastre. La mise en place du cadastre, aujourd'hui absent, prévue pour 2014, sera aussi une grande nouveauté dans une île où il n'a jamais existé avec les problèmes que l'on peut imaginer pour réussir à le mettre en oeuvre.

Un des enjeux politiques majeurs de la départementalisation concerne les solutions à apporter à l'immigration clandestine, du point de vue des autorités françaises, des habitants de l'Union des Comores vers Mayotte. Selon l'Insee, $40 \%$ de la population de Mayotte n'est pas de nationalité française (Insee, 2009). Cela revient à dire que près de 76000 habitants sur les 187000 que compte Mayotte sont soit nés à l'étranger (les deux tiers) soit nés sur l'île mais de parents étrangers (un tiers). Il faut préciser qu'environ $96 \%$ des étrangers à Mayotte sont Comoriens et que les $4 \%$ restant sont majoritairement constitués par des Malgaches. Le nombre d'expulsés est passé d'environ 8000 en 2004 à plus de 16000 en 2008. Ce chiffre est sensiblement équivalent à la moitié des reconduites à la frontière pratiquée en France. Ces retours au point de départ s'accompagnent depuis plusieurs mois, et notamment depuis la vidéo tournée par un agent de la Police aux frontières (PAF) et visible sur le site Internet du journal Libération (cf. Libération, 2008), d'une mobilisation de l'opinion publique nationale française et des médias nationaux et internationaux pour dénoncer une situation peu conforme aux respects des droits de l'homme constatée au centre de rétention de Mayotte situé à quelques encablures de l'aéroport de Pamandzi (figure 2) sur Petite Terre.

Figure 2 - L'archipel de Mayotte

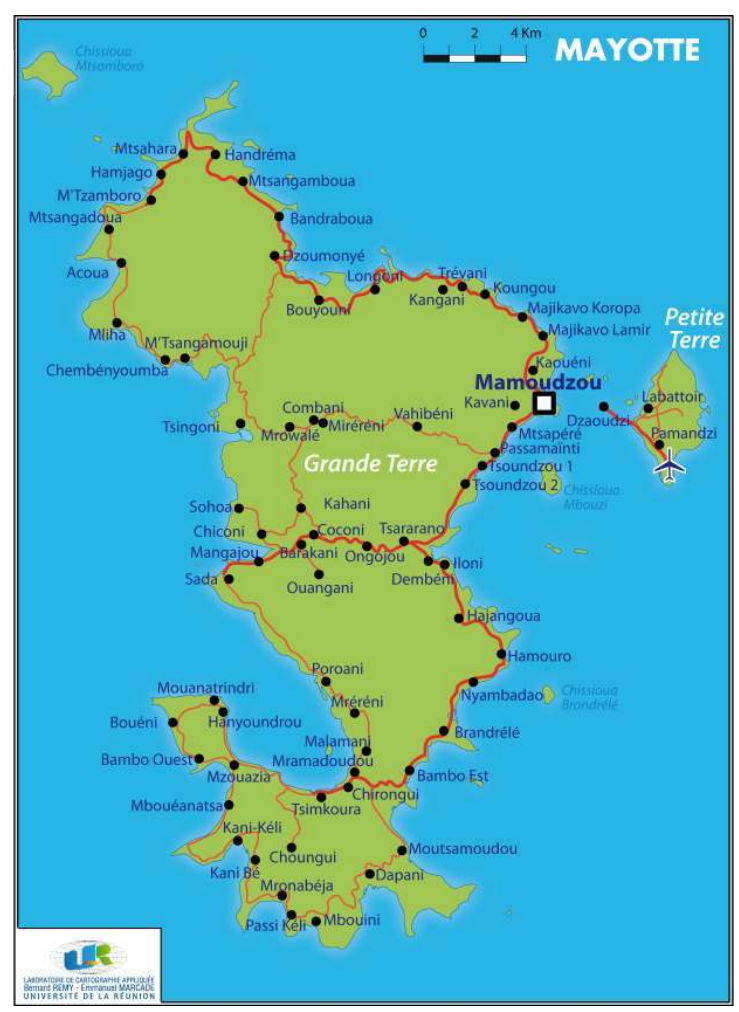



de la classe politique mahoraise et du peuple mahorais que d'avoir mené un combat de l'île de Mayotte dans le giron de l'Union des Comores. De fait, depuis son indépendance en 1975, l'Union des Comores revendique toujours Mayotte à la France et dénonce de concert avec l'ONU une décolonisation inachevée. Dans cette logique, le gouvernement de l'Union des Comores considère le référendum du 29 mars 2009 comme «nul et non avenu et sans effet » (AFP cité par La lettre de Malango du 31 mars 2009). Ainsi, le président comorien Ahmed Abdallah Sambi tente de mobiliser les pays de la Ligue arabe pour qu'ils expriment dans les tribunes internationales «leur solidarité avec le peuple des îles Comores dans la défense de son droit légitime, juridique et souverain à recouvrer l'île de Mayotte " (AFP cité par Le Monde du 30 mars 2009). Dans le même temps, L'Union des Comores doit faire face à une instabilité politique chronique et à la tentation du séparatisme qui est grande entre les trois îles, Anjouan, Grande Comore et Mohéli (Taglioni, 2008). L'agitation politique et populaire qui a précédé le référendum le 17 mai 2009 sur la révision constitutionnelle dans l'Union (pour notamment réduire le pouvoir des présidents des trois îles, harmoniser la durée des mandats et le calendrier des diverses élections et redéfinir la présidence tournante entre les îles au péril de la présidence prochaine de Mohéli prévue en 2010) reflète bien la fragilité de l'union politique aux Comores. Aux Comores, comme à Mayotte, c'est bien le oui qui l'a emporté dans ce référendum constitutionnel avec près de $94 \%$ des votes. Le gouvernement central reprend donc l'initiative au détriment des pouvoirs locaux de chacune des trois îles, le président Sambi voit son mandat prolonger d'un an et l'islam pourrait à nouveau devenir religion d'Etat comme à l'époque de la République fédérale islamique des Comores.

21 On le voit, les défis sont grands pour Mayotte et à ce jour, il est vraisemblable que la départementalisation, en tant que moteur de développement et de levier pour le rattrapage socio-économique de Mayotte, ne sera pas une réalité, autre qu'institutionnelle, pour les Mahorais avant deux ou trois décennies. L'intégration politique de Mayotte à la république française, et plus encore à l'Union européenne avec un statut de région ultrapériphérique (RUP) pour le moment très hypothétique bien que théoriquement envisageable à partir de 2014, ne résoudra donc pas d'un coup d'un seul les problèmes bien spécifiques que connaissent les Mahorais. D'autant que le problème crucial de l'engagement financier de l'Etat pour l'accompagnement des Mahorais dans la départementalisation n'est pas encore clairement établi. Un fonds de développement 
économique, social et culturel a bien été évoqué dans le pacte pour la départementalisation mais, rien n'est budgété pour le moment.

A titre de comparaison, les exemples de La Réunion ou encore des départements français d'Amérique (DFA) sont éloquents puisque plus de 60 ans après leur départementalisation, en 1946, ces territoires connaissent encore un retard économique sensible par rapport à la métropole. Cette fragilité économique se double d'une agitation sociale (Daniel, 2009) et identitaire récurrente qui s'est à nouveau exprimée en ce début d'année 2009 en particulier dans les Antilles françaises avec comme conséquence possible une évolution statutaire dans les années à venir.

\section{L'instabilité de l'outre-mer : une spécificité française?}

On peut se poser la question de la spécificité de l'instabilité institutionnelle, politique et sociale des outre-mers français dans le paysage mondial. De fait, de nombreux Etats dans le monde ont des liens institutionnels plus ou moins formels avec des territoires, plus précisément des micro-territoires. L'expression de micro-territoire, pour la plupart insulaire, désigne des espaces qui dépendent, à divers degrés, d'une métropole souvent fort éloignée. On remarquera du reste qu'il n'existe pratiquement aucun territoire continental appartenant à une métropole sans y être territorialement rattaché. Ces micro-territoires insulaires sont en grande majorité les expressions ultimes des empires coloniaux. Ces associats sont aujourd'hui dispersés sur tous les océans et mers du monde. Les mères-patries sont notamment européennes (Danemark, Espagne, France, Pays-Bas, Portugal, Royaume-Uni), chilienne, étatsunienne ou néo-zélandaise. Ces micro-territoires ont des statuts fort différents au regard des droits nationaux, communautaires ou internationaux mais peu connaissent les tourments politico socio-économiques des outremers français.

En ce qui concerne les outre-mers du Royaume-Uni, c'est le statut de colonie qui est toujours en place. Les colonies sont néanmoins largement autonomes et elles ont chacune un conseil exécutif et un parlement législatif. Le Royaume-Uni tente de rationaliser et de moderniser la gestion de ces territoires depuis plusieurs décennies avec plus ou moins de réussite. Ce statut est à rapprocher de celui de l'île de Tokelau qui est administrée par la Nouvelle-Zélande. Les Antilles néerlandaises forment une région autonome du Royaume tripartite des Pays-Bas. Aruba est la deuxième composante du Royaume et les Pays-Bas la troisième. Néanmoins, la fédération des Antilles néerlandaises, actuellement constituée de 5 îles, s'acheminent vers une dissolution avec la mise en place prochainement de 2 nouveaux Etats fédéraux (Curaçao et Sint-Maarten) sur le modèle d'Aruba et de 3 nouvelles communes du Royaume des Pays-Bas. Le cas du Royaume des Pays-Bas est intéressant car il pointe la difficulté pour des entités politiques multi-insulaires de résister à la tentation du séparatisme ou de l'autonomie et cela parfois même au-delà de la difficulté à composer avec une puissance tutélaire (Taglioni, 2005). Les territoires américains sont membres du Commonwealth des États-Unis. L'exécutif est aux mains d'un gouverneur élu au suffrage universel et le législatif est assuré par un sénat, élu, également, au suffrage universel. Ce qui ne veut pas dire pour autant qu'il n'y a pas de problème de fond pour ces territoires américains. Le cas de Porto Rico est éloquent à ce sujet puisque l'insatisfaction vis-à-vis du statut de l'île est sujet de crispation politique et crescendo ces dernières années. Pour l'Espagne, Baléares et Canaries sont des communautés autonomes au même titre que les 15 autres que compte le pays comme 
l'Andalousie, la Catalogne, la Galice, La Rioja etc. Pour les Açores et Madère, c'est le statut de région autonome de la République du Portugal qui prévaut avec pour chaque région des assemblées législatives et exécutives. Le Groenland et les îles Féroé sont deux régions autonomes du Danemark avec néanmoins pour la première un approfondissement de l'autonomie depuis juin 2008 et une possible indépendance dans les années à venir. L'Île de Pâques et l'archipel Juan Fernández peuvent être considérés comme des outre-mers du Chili. Ce sont des provinces de la région de Valparaíso.

A la lumière de ces quelques exemples, il ressort que les outre-mers, autres que français, que l'on vient de décrire sont tous dans des situations d'autonomie plus ou moins affirmée. Certes les relations avec la métropole ou encore au sein des archipels sont souvent complexes voire parfois tendues mais néanmoins, l'autonomie du législatif et de l'exécutif semble la condition a minima d'une possible gouvernance dans des espaces périphériques à leur métropole. C'est peut-être ce qui manque aux outre-mers français qui n'ont pas encore atteint pour certains ce niveau décisionnel, c'est le cas des départements d'outre-mer, pour pouvoir se positionner dans une relation apaisée avec les autorités françaises. D'ailleurs si aujourd'hui la Nouvelle-Calédonie ou encore la Polynésie française entretiennent des relations moins passionnées avec le gouvernement français c'est parce que ces deux collectivités ont accédé à un degré d'autonomie avancé et en devenir qui tempère les revendications et les blessures profondes du passé. La souveraineté partagée présente sans doute une alternative précieuse entre souveraineté et dépendance (Taglioni, 2006).

Par ailleurs, la multiplicité des statuts institutionnels des outre-mers français est sans doute un élément qui brouille la donne d'une bonne gouvernance car vouloir adapter la République à chaque spécificité territoriale c'est diluer ensuite la portée des politiques publiques de développement humain. Durant les états généraux de l'outre-mer, qui se déroulent avec un succès très relatif en ce moment, sans doute cette question de la prolifération des statuts sera-t-elle examinée avec attention tant elle est porteuse aussi bien de solutions que de problèmes.

Dans ce contexte, la départementalisation de Mayotte constitue un vrai paradoxe de l'histoire des nations, qui tendent toutes a minima vers l'autonomie, et on peut s'interroger sur la durabilité de ce statut de département d'outre-mer au sein de la république française qui va à l'encontre de l'aspiration libertaire des élus martiniquais ou guyanais et plus généralement des autres outre-mers de par le monde qui, en dépit d'une décolonisation inachevée d'hier, inventent et proposent de nouveaux modes de gouvernance aujourd'hui.

\section{BIBLIOGRAPHIE}

Agniel, G. ; Faberon, J.-Y. (dir.), 1999. La souveraineté partagée en Nouvelle-Calédonie et en droit comparé. Paris, La documentation française, $180 \mathrm{p}$. 
Bonnelle, F., 1998. Réflexions sur l'avenir institutionnel de Mayotte. Paris, La documentation française, $128 \mathrm{p}$.

Daniel, J., 2009. La crise sociale aux Antilles. Echogéo, Sur le vif 2009

http://echogeo.revues.org/index11117.html

Faberon, J.-Y. (dir.), 2006. Les collectivités françaises d'Amérique au carrefour des institutions. Paris, La documentation française, $368 \mathrm{p}$.

Gay, J-Ch., 2005. "Où en est la France d'outre-mer ?". MappeMonde, $\mathrm{n}^{\circ} 79$

http://mappemonde.mgm.fr/num7/lieux/lieux05301.html

La Documentation française, 2008. Quelles sont les collectivités territoriales situées en outre-mer ? Mise à jour le 11 juillet 2008.

http://www.vie-publique.fr/decouverte-institutions/institutions/collectivites-territoriales/ outre-mer/existe-t-il-differents-types-collectivites-outre-mer.html\#

Laïdi, Z., 2003. « Vers un monde multipolaire ». Etudes, n 10, p. 297-310

La lettre de Malango $n^{\circ} 110,31$ mars 2009

http://www.malango-actualite.com/lettre/lettre-110.htm

Libération, 2008. « Mayotte : le centre de rétention, uen zone de non-droit », Libération, $\mathrm{n}^{\circ} 8591$, 18 décembre 2008 , p. 2-5

Michalon, T., 2009. L'outre-mer français. Evolution institutionnelle et affirmations identitaires. Paris, l'Harmattan, $164 \mathrm{p}$.

Pacte pour la départementalisation de Mayotte http://www.departementdemayotte.com/index.php? option=com_content\&view=article\&id=57\&Itemid $=63$

Souef, M., 2009. Les grands défis de la politique étrangère des Comores. Levallois Perret, Editions de la lune, $134 \mathrm{p}$.

Taglioni, 2008. « L'île d'Anjouan figure de la balkanisation de l'archipel des Comores ». Revue EchoGéo, CNRS, rubrique « Sur le vif » 2008

http://echogeo.revues.org/index7223.html

Taglioni, F., 2006. « Les petits espaces insulaires face à la variabilité de leur insularité et de leur statut politique ». Les Annales de géographie, n652, p. 664-687

Taglioni, F., 2005. « Les revendications séparatistes et autonomistes au sein des États et territoires mono- et multi-insulaires. Essai de typologie ». Cahiers de géographie du Québec, n¹36, p. $5-18$

http://www.cgq.ulaval.ca/textes/vol_49/no136/03-Taglioni.pdf

\section{RÉSUMÉS}

Le champ géographique des compétences de l'administration territoriale française va évoluer en 2011 avec la création du $101^{\mathrm{e}}$ département, celui de Mayotte. Cette départementalisation hors de l'hexagone est le fruit d'un long processus politique commencé en 1975 à la date du premier référendum qui allait par la suite séparer Mayotte du reste de l'archipel des Comores. Cette départementalisation s'inscrit dans un climat d'instabilité sociale et politique particulièrement aigu dans l'ensemble des quatre départements français d'outre-mer depuis plusieurs mois. Elle apparaît ainsi comme un paradoxe dans l'histoire des outre-mers français, et des outre-mers 
d'autres pays dans le monde, qui cherchent en général à s'affranchir de la puissance tutélaire au lieu d'y être assimilés.

The geographical field of the French administration jurisdiction will evolve in 2011 with the creation of the 101st department: Mayotte. This departmentalization outside the hexagon is the result of a long political process begun in 1975 to the date of the first referendum that would thereafter Mayotte separate from the rest of the Comoros archipelago. This departmentalization comes within a social and political instability particularly acute for several months in the French overseas departments. It appears as a paradox in the history of the French overseas, and overseas from other countries in the world, which generally seek to escape the tutelary power instead of being assimilated.

\section{INDEX}

Keywords : French overseas territories, Mayotte, Guadeloupe, Martinique, Reunion Island, French Guyana, political integration, political autonomy, territorial claims

Mots-clés : Outre-mer français, Mayotte, Guadeloupe, Martinique, La Réunion, La Guyane, évolution statutaire, intégration politique, autonomie politique, revendication territoriale

\section{AUTEUR}

\section{FRANÇOIS TAGLIONI}

François Taglioni (tagli@diplomacy.edu) est directeur de recherche à l'IRD, UR 029, en affectation à La Réunion et membre de l'UMR PRODIG. Spécialiste des petits espaces insulaires en développement dans le monde, il travaille actuellement plus particulièrement sur les interactions santé/environnement dans le sud-ouest de l'océan Indien.

Site Internet de l'auteur : http://www.taglioni.net 